EchoGéo

\title{
Acquisitions de la Bibliothèque Géographie
}

\section{Bernadette Joseph}

\section{(2) OpenEdition}

Journals

Édition électronique

URL : https://journals.openedition.org/echogeo/2200

DOI : $10.4000 /$ echogeo.2200

ISSN : 1963-1197

\section{Éditeur}

Pôle de recherche pour l'organisation et la diffusion de l'information géographique (CNRS UMR 8586)

\section{Référence électronique}

Bernadette Joseph, «Acquisitions de la Bibliothèque Géographie », EchoGéo [En ligne], 4 | 2008, mis en ligne le 05 mars 2008, consulté le 31 juillet 2021. URL : http://journals.openedition.org/echogeo/2200 ; DOI : https://doi.org/10.4000/echogeo.2200

Ce document a été généré automatiquement le 31 juillet 2021.

EchoGéo est mis à disposition selon les termes de la licence Creative Commons Attribution - Pas d'Utilisation Commerciale - Pas de Modification 4.0 International (CC BY-NC-ND) 


\title{
Acquisitions de la Bibliothèque Géographie
}

\author{
Bernadette Joseph
}

Afrique

1 Angola : oil, broad-based growth, and equity, Washington, D.C., World Bank, cop. 2007, G 19-3337

BATTISTINI, René, Géographie de l'Afrique orientale, Kenya, Uganda et Tanzanie, Paris, Centre de documentation universitaire, 1969, BCOURS 314 BEN YAHMED, Gabon, un pari d'avenir, Paris, les Éd. du Jaguar, DL 2007, G 19-3336

BEN YAHMED, HOUSTIN, NDIAYE, WADE, Atlas du Sénégal, Paris, les Éd. J.A., impr. 2007, G 19-3353

CHARTON, H., L'Afrique orientale : annuaire 2005, G 19-3289 IOP, NIANG DIENE, BARRY, SANÉ, Les États-nations face à l'intégration régionale en Afrique de l'Ouest, Paris, Karthala, DL 2007, G 19-3344 Diversité et culture, Paris, CulturesFrance, DL 2007, G 19-3338 FERRY, L'Afrique face à ses défis démographiques : un avenir incertain, Paris ; Nogent-surMarne, Agence française de développement : Kathala: Centre population et développement, 2007, G 19-3351 HOMBERT, PERROIS, Coeur d'Afrique : Gorilles, Cannibales et Pygmées dans le Gabon de Paul Du Chaillu, Paris, CNRS éditions, impr. 2007, G 19-3354

10 JACOB, Jean-Pierre, Terres privées terres communes : gouvernement de la nature et des hommes en pays winye (Burkina Faso), Paris, IRD éditions, 2007, G 19-3349

11 KASONGO-NUMBI, Kashemukunda, Les eaux et forêts de la RD Congo : les enjeux... : changement climatique de la planète, Paris, l'Harmattan, DL 2007, G 19-3345 MARIE, Jérôme, Avenir du fleuve Niger, Paris, IRD, 2007, G 19-3341

13 MARYSSE, S., L'Afrique des Grands Lacs : annuaire 2006-2007, G 19-3352 
MOINE, Alexandre, Le territoire : comment observer un système complexe, Paris, l'Harmattan, DL 2007, G 19-6592

PARIS, Henri, Le pétrole tue l'Afrique, Paris, les Ed. des Riaux, cop. 2007, G 19-3342

RAJAONAH, BOIS, Marchés urbains de Madagascar XIXe, XXe siècles : Antananarivo, Antsirabe, Antsiranana, Paris, l'Harmattan, DL 2007, G 19-3343

SOUMAH, Ibrahima, Avenir de l'industrie minière en Guinée, Paris, l'Harmattan, DL 2007, G 19-3340

8 VELLUT, Villes d'Afrique : explorations en histoire urbaine, Tervuren (Belgique) ; Louvainla-Neuve ; Paris, Koninklijk museum voor Midden-Afrika = Musée royal de l'Afrique centrale : Université catholique de Louvain, Chaire d'études africaines : Éd. l'Harmattan [etc.], 2007, G 19-3346

WAZIRI MATO, SANÉ, BARRY, Les États-nations face à l'intégration régionale en Afrique de l'Ouest : le cas du Niger, Paris, Karthala, DL 2007, G 19-3339

Amérique du nord

CANOBBIO, Eric, BOISSIÈRE, Atlas des pôles : régions polaires : questions sur un avenir incertain, Paris, Éd. Autrement, impr. 2007, G 20-1600

Developing retail entertainment destinations, Washington, Urban Land Institute, cop. 2001, G 20-1597

DOUZET, Frédérick, La couleur du pouvoir : géopolitique de l'immigration et de la ségrégation à Oakland, Californie, Paris, Belin, DL 2007, G 20-1595

FOCH, René, La haute autorité de la vallée du Tennessee: Tennessee valley authority, Paris, Presses universitaires de France, 1952, G 20-1598

ILLINGWORTH, Frank, ALLAIN, La vie au nord du Cercle polaire arctique, Paris, Payot, 1954, G 20-1599

MAUMI, Catherine, Thomas Jefferson et le projet du Nouveau monde, Paris, Éd. de la Villette, DL 2007, G 20-1596

Amérique Latine

ANICETO BLANCO, Pedro, Diccionario geográfico del departamento de Oruro, Lima ; La Paz ; [Stockholm], IFEA : IEB, Instituto de estudios bolivianos : Asdi, DL 2006, G 21-1633

AZAN, Paul, Argentine : terre promise, Paris, Hachette, 1943, G 21-1639

BOHOU, dit Zouzougbo Jean-Marie, COLLIN DELAVAUD, Géopolitique et projection de puissance du Brésil au XXIe siècle, Paris, L'Harmattan, cop. 2007, G 21-1628

BOTTON, Sarah, La multinationale et le bidonville : privatisations et pauvreté à Buenos Aires, Paris, Karthala, impr. 2007, G 21-1630

DELER, Jean-Paul, Genèse de l'espace équatorien : essai sur le territoire et la formation de l'État national, Lima ; Paris, Institut français d'études andines : Éditions A.D.P.F, 1981, G 21-830

1 DURIZOT JNO-BAPTISTE, YACOU, Les risques majeurs aux Antilles : approche culturelle et prévention sociale, Paris, CERC - Université des Antilles et de la Guyane : Karthala, impr. 2007, G 21-1625

FERNÁNDEZ CHRISTLIEB, GARCÍA ZAMBRANO, Territorialidad y paisaje en el Altepetl del siglo XVI, Mexico, Fondo de Cultura Económica : Instituto de Geografía de la Universidad Nacional Autónoma de México, cop. 2006, G 21-1629 


\section{Atlas}

Asie

MILES, Mike E., BERENS, WEISS, Real estate development : principles and process, Washington, Urban Land Institute, c2000, G 21-1635

SABOURIN, Eric, HAUBERT, Paysans du Brésil : entre échange marchand et réciprocité, Versailles, Editions Quae, 2007, G 21-1632

SALAVERRY, José A., Macro-ecología de los Andes peruanos : situación actual y dynámica de cambio en los últimos 20000 años, Lima, CONCYTEC, Consejo nacional de ciencia, tecnología e innovación tecnológica : IFEA, Institut francais d'études andines, 2006, G 211637

SCHMITZ, Adrienne, Real estate market analysis : a case study approach, Washington, D.C., Urban Land Institute, c2001, G 21-1636

VICTOR, Paul-Émile, Adieu l'Antarctique, Paris, Robert Laffont, impr. 2007, G 21-1627

BERNOT, Lucien, Les paysans Arakanais du Pakistan oriental : l'histoire, le monde végétal et l'organisation sociale des réfugiés Marma (Mog), Paris ; La Haye, Mouton, 1967, G 17-907-<1>

GotTMAnN, Jean, Etudes sur l'état d'Israël et le Moyen-Orient, Paris, A. Colin, 1959, G 17-375

GURBANOV, Turab, Le pétrole de la Caspienne et la politique extérieure de l'Azerbaïdjan, Paris, L'Harmattan, DL 2007, G 17-2602-<1>

GURBANOV, Turab, Le pétrole de la Caspienne et la politique extérieure de l'Azerbaïdjan, Paris, L'Harmattan, DL 2007, G 17-2602-<2>

Historic conservation policies in Seoul, Beijing and Tokyo, Seoul, Seoul Development Institute, 2005, G 17-2609

KERBE, Jehad, Climat, hydrologie et aménagements hydro-agricoles de Syrie, Lille ; Talence, Atelier national de reproduction des thèses : diff. Presses universitaires de Bordeaux, 1987, G 17-1584-<1>

KONINCK, Rodolphe de, Malaysia : la dualité territoriale, Paris, Éd. Belin : la Documentation française, DL 2007, G 17-2598

LARIVIÈRE, La Chine et les Chinois de la diaspora, Paris, CNED-SEDES, 1999, A 17.4 46

RAILLON, François, Indonésie : les voies de la survie, Paris, Ed. Belin : la Documentation française, DL 2007, G 17-2599

RECLUS, Élisée, L'empire du milieu : le climat, le sol, les races, la richesse de la Chine, Paris, Editions You-Feng, impr. 2007, G 17-2605

ROBEQUAIN, Charles, L'Indochine française, Paris, A. Colin, 1948, G 17-2607

SABOURET, Jean-François, FILLON, L'empire de l'intelligence : politiques scientifiques et technologiques au Japon depuis 1945, Paris, CNRS éditions, DL 2007, G 17-2604

SHAW, Wendy S., Cities of whiteness, Malden, Blackwell publ., cop. 2007, G 17-2603

WILlOQUET, Gaston, Histoire des Philippines, Paris, Presses universitaires de France, 1961, K 1055

Atlas del Golfo de Urabá : una mirada al Caribe de Antioquia y Chocó, Santa Marta, Invemar, 2007, AQ 21-42 

entreprises, régulations et trajectoires : actes du colloque international de Besançon, 27, 28 et 29 octobre 2004, [Besançon], Presses universitaires de Franche-Comté, 2007, G 3-2901

Atlas transfrontalier, Lille, Région Nord-Pas de Calais : Direction régionale de l'équipement du Nord-Pas-de-Calais : Direction régionale de l' Insee Nord-Pas-de-Calais, DL 2006, AQ 15-143-<4>

Atlas transfrontalier, Lille, Région Nord-Pas de Calais : Direction régionale de l'équipement du Nord-Pas-de-Calais : Direction régionale de l' Insee Nord-Pas-de-Calais, DL 2006, AQ 15-143-<5>

Carta dei tipi d'impresa nell' agricultura Italiana, Roma, 1958, AQ 15-154

Cartographie des pays du Sahel, [Paris], Ministère de la coopération, cop. 1976, AQ 19-57

CATTANEO, Marco, Le tour du monde des plus belles villes, Vercelli ; Paris, Éd. White star, DL 2007, AQ 9-44

DRESCH, Nouvel atlas des formes du relief, [Paris], F. Nathan, 1985, AF 8-44

Geográfia de Centroamérica y del Caribe, Tegucigalpa, Ed. Ramses, 2006, AQ 21-41

HALL, Atlas of South-east Asia, London ; New York, Macmillan : St Martin's Press, 1964, AQ 17-76

JENSEN, Ruth Helkiaer, Topografisk atlas Danmark : . Redigeret af Ruth Helkiaer Jensen og Kr. Marius Jensen, Københaven, Det Kongelige Danske Geografiske Selskab, 1976, AQ 15-156

JOHNSTON, Andrew K., La terre vue de l'espace, [Paris], Viamédias, DL 2006, AQ 9-43

L'Atlas des atlas : frontières, conflits, idéologies, prospectives, utopies, Paris, Courrier international, 2005, AQ 5-155

L'Atlas National Geographic, Washington, National Geographic Society, cop. 2007, AQ 5-154

Le grand atlas géographique et encyclopédique du monde, Paris, Éd. Atlas, impr. 2007, AQ 9-45

ZAREMBY, Atlas ziem odzyskanych = Atlas des territoires récupérés de la Pologne..., Warszawa, Glowny urzad planowania przestrzenne go, 1947, AF 15-68

Congrès

Action publique et changements d'échelles : les nouvelles focales du politique, Paris, l'Harmattan, DL 2007, G 3-2905

BEN BOUBAKER, XXème colloque international de climatologie : Climat, tourisme, environnement : actes du colloque de Carthage (Tunisie), CENAFFE, 3-8 septembre 2007, Tunis, Université de Tunis : Université de Manouba, cop. 2007, G 3-2898

BLETON-RUGET, POIRRIER, Le temps des sciences humaines : Gaston Roupnel et les années trente, Paris, Le Manuscrit, cop. 2006, G 3-2899

CORVOL, Forêt et eau : XIIIe-XXIe siècle, Paris, l'Harmattan, DL 2007, G 3-2909

DAUMAS, LAMARD, TISSOT, Les territoires de l'industrie en Europe, 1750-2000 :

FAURE, NÉGRIER, Les politiques publiques à l'épreuve de l'action locale : critiques de la territorialisation, Paris, l'Harmattan, DL 2007, G 3-2903

HOCHART, Architecture et urbanisme commercial : [colloque organisé à l'Ecole d'architecture de Lille le 15 mai 1998], [Villeneuve d'Ascq], Ecole d'architecture de Lille, Régions Nord, [2000], G 3-2896 

Royaume-Uni, Suède, Bulgarie, Estonie, Hongrie, Lettonie, Lituanie, Pologne, Roumanie, Slovaquie, Tchéquie : Germany, Italy, Spain, Sweden, United Kingdom, Bulgaria, Czech Republic, Estonia, Hungary, Latvia, Lithuania, Poland, Romania, Slovakia : dited by Jean-Claude Boual, Philippe Brachet and Malgorzata Hiszka, Paris, Publisud, [2007], G 15-5687 
CABOURET, Michel, La Finlande, Paris, Éd. Karthala, impr. 2005, G 15-5347

CHRISTALLER, Walter, BASKIN, Central places in Southern Germany, Englewood Cliffs (New Jersey), Prentice-Hall, [cop. 1966], G 15-1357

CIATTONI, La Russie, Paris, Éd. Sedes, impr. 2007, G 15-5660

Città, commercio e grandi eventi, Torino, CELID, impr. 2005, G 15-5716

DAUMAS, Max, BENNASSAR, Un géographe dans les Pyrénées aragonaises, Pau, Ed. Cairn, DL 2007, G 15-5577

DEMANGEON, Albert, Le Rhin : problèmes d'histoire et d'économie, Paris, A. Colin, 1935, G 15-1

DEMANGEON, Albert, PERPILLOU, Le Déclin de l'Europe, Paris, Librairie Guénégaud, 1975, G 15-2460

DOLLFUS, Jean, L'homme et le Rhin, Paris, Gallimard, 1960, G 15-714

DUMONT, Isabelle, HÉRIN, Tapecul, squat, soleil : vivre la détresse sociale dans le centre-ville : essai de géographie sociale, Caen ; [Mont-Saint-Aignan], Pôle universitaire normand : Presses universitaires de Caen : Publications des universités de Rouen et du Havre, 2007, G 15-5688

ESPOSITO, Marie-Claude, Le renouveau de l'économie britannique, Paris, Economica, DL 2007, G 15-5686

FARAMOND, GLAYMAN, Suède : la réforme permanente, Paris, Stock, DL 1977, G 155700

FARAMOND, Guy de, La Suède et la qualité de la vie, [Paris], le Centurion, 1975, G 155699

FILIPPUCCI, Mutamenti nella geografia dell'economia italiana, Milano, F. Angeli, cop. 2006, G 15-5696

GERBET, Pierre, La construction de l'Europe, Paris, A. Colin, DL 2007, G 15-5674

HEPPELL, Muriel, Yugoslavia, London, E.Benn, 61, G 15-5705

HERSLUND, Lise, Rural diversification and change in the Baltic countryside : rural inhabitants and business in Latvia and Estonia : a local perspective, København, Geografisk Institut, Københavns Universitet, cop. 2007, G 15-5675

KERJEAN, Alain, BRUTARU, GHEORGHIU, Voyage en Roumanie de la Transylvanie au delta du Danube, Grenoble ; [Paris], Glénat : La société de géographie, impr. 2007, G 155693

KOLOSSOV, La Russie : espaces, villes, réseaux, Nantes, Editions du Temps, impr. 2007, G 15-5684

KOLOSSOV, La Russie : espaces, villes, réseaux, Nantes, Editions du Temps, impr. 2007, G 15-5684

La Russie, Paris, La Documentation française, 2007, G 15-5714

1 LAMARD, WOESSNER, Rhin-sud, un territoire en devenir ?, Belfort, Université de technologie de Belfort-Montbéliard, 2007, G 15-5673

2 LEVITT, Property development : Europe : case studies in innovation, Washington, Urban Land Institute, c2003, G 15-5697

MARCHAND, Pascal, Atlas géopolitique de la Russie : puissance d'hier, puissance de demain ?, Paris, Éd. Autrement, impr. 2007, G 15-5676

MASBOUNGI, GRAVELAINE, ISHERWOOD, Birmingham : faire la ville en partenariat, Paris, Ed. de la Villette, DL 2006, G 15-5534

EchoGéo, 4 | 2008 
MEAD, W. R., Farming in Finland, [London], University of London, Athlone Press, 1953, G 15-5704

MERRIMAN, Peter, Driving spaces : a cultural-historical geography of England's M1 Motorway, Malden ; Oxford, Blackwell publ., cop. 2007, G 15-5666

PARANQUE, GRENIER, LEVRATTO, L'Euro-Méditerranée : de l'espace géographique aux modes de coordination socio-économiques, Paris, l'Harmattan, DL 2007, G 15-5681

PARENT, Jean, Le Modèle suédois, Paris, Calmann-Lévy, 1970, G 15-5698

PETITEVILLE, Franck, La politique internationale de l'Union européenne, Paris, Presses de Sciences Po, DL 2006, G 15-5664

RADVANYI, Jean, La nouvelle Russie, Paris, A. Colin, impr. 2007, G 15-5668

REITEL, François, Mers et fleuves : variations sur le thème de l'eau, Paris, Didier, 1973, G 15-2122

REY, GROZA, IANO?S, P?TROESCU, Atlas de la Roumanie, Montpellier ; Paris, CNRS-GDRE S4 : La Documentation française, 2007, G 15-5682

ROTHERY, Agnès, Le Danemark dans le monde, Paris, Payot, 1938, G 15-5702

ROULLEAU-BERGER, Laurence, Nouvelles migrations chinoises et travail en Europe, Toulouse, Presses universitaires du Mirail, impr. 2007, G 15-5679

SPAGNOLI, Luisa, Tor Vergata : continuità e modificazioni nel paesaggio della campagna romana, Roma, Società geografica italiana, 2006, G 15-5690

STREYFFERT, Thorsten, Les Forêts et les industries forestieres de la Suède, Paris, BergerLevrault, 1939, G 15-5701

TANNER, Rolph Peter, Geopolitische Dynamik und Verkehr im Fürstbistum Basel von der Antike bis zum Eisenbahnbau : "Die Bistums-Lande, wie bekant, rauch, ohnwegsam, mithin zum Commercio auf keine Weiss bequemlich“, Bern, Geographisches Institut des Universität, cop. 2007, G 15-5667

TARRIUS, Alain, La remontée des Sud: Afghans et Marocains en Europe méridionale, La Tour d'Aigues, Ed. de l'Aube, impr. 2007, G 15-5680

THOREZ, La Russie, Paris, CNED : Éd. Sedes, impr. 2007, G 15-5670

VINATIER, Laurent, ROY, Russie : l'impasse tchétchène, Paris, A. Colin, impr. 2007, G 15-5663

WACKERMANN, La Russie en dissertations corrigées, Paris, Ellipses, impr. 2007, G 155659

Welt der Alpen, Erbe der Welt : UNESCO Welterbe-Region Jungfrau-AletschBietschhorn, Bern, Haupt Verl., cop. 2007, G 15-5689

ZORGBIBE, Charles, PINTO, Les Etats-unis scandinaves, Paris, Éditions Pédone, 1968, G 15-5703

Fonds russe

ALEKSANDROV, Sergeï Mihaïlovič, Ostrov Sahalin, Moskva, Nauka, 1973, R 833

ANUČIN, Regional'noe razvitie i geografičeskaâ sreda, Moskva, Akademiâ nauk SSSR, 1971, R 911

BABAEV, Opyt izučeniâ i osvoeniâ Vostočnyh Karakumov : Sbornik, posvâsennyï 60letiû Repetekskoï pesčano-pustynnoï stancii, Ašhabad, izdatel'stvo Ylym, 1972, R 825 
BAŽENINA, Geomorfologičeskoe kartografirovanie v s"emočnyh masštabah, [Moskva], Moskovskiï universitet, 1975, R 909

ČEMEKOV, Û.F., Metodičeskoe rukovodstvo po geomorfologičeskim issledovaniâm, Leningrad, Nedra, 1972, R 860

General physical geography, Moscou, [s.n.], 1976, R 1001

Geografiâ proizvoditel'nyh sil Severnogo Kazahstana : Tom 1 Prirodnye usloviâ i resursy, [Moskva], izd-vo Moskovskogo universiteta, 1972, R 861

Geologičeskie formacii Zapadnogo Predkavkaz'â, Moskva, Nauka, 1973, R 857

GERKE, Problemy sistematiki spiral'nykh nodozariid, Vil'nius, Mintis, 1975, R 900

GRIGÂLIS, Fauna i stratigrafiâ paleozoâ i mezozoâ Pribaltiki i Belorussii : [sbornik statei], Vilnius, Mintis, 1975, R 905

GURŽIÏ, Ukraïnskiï istoriko-geografičniï zbirnik : vypusk 2, Kiïv, Naukova dumka, 1972, R 910

KOMAROV, Vasiliï Efimovič, Dohody i potreblenie naseleniâ SSSR, Moskva, Nauka, 1973, R 854

KOVALEV, Evgeniï Vladimirovič, Agrarnye reformy v stranah Latinskoï Ameriki, Moskva, Nauka, 1973, R 855

KOZLOV, Igor' Dmitrievič, Sotrudničestvo stran - členov SÈV v ènergetike, Moskva, Nauka, 1973, R 856

KUPERMAN, Biologičeskie osobennosti i usloviâ proizrastaniâ sel'skohozâistvennyh kul'tur v Altaïskom krae, [Moskva], Moskovskij universitet, 1974, R 907

MARINOV, Gidrogeologiâ Azii, Moskva, Nedra, 1974, R 853

Narodnoe hozâjstvo SSSR v 1973 g. : statisticeskij ežegodnik, Moskva, Statistika, 1974, R 850

OL'DEROGGE, The Countries and peoples of the East : selected articles, Moscow, Nauka Publishing House, Central Department of Oriental Literature, 1974, R 919

Resursy sreda rasselenie, Moskva, Nauka, 1974, R 859

ROZDESTVENSKIÏ, Aleksandr Petrovic, Noveišaâ tektonika i razvitie rel'efa Ûžnogo Priural'â, Moskva, Nauka, 1971, R 94

Severnyï Kavkaz, Zakavkaz'e : Putevoditel' èkskursii dlâ u?astnikov kongressa, Moscou, [s.n.], 1976, R 679

ŠIRINOV, Naib Š., Noveiišaâ tektonika i razvitie rel'efa Kura-Araksinskoï depressii, Baku, Èlm, 1975, R 941

Stratigrafiâ, sedimentologiâ i geologiâ četvertičnogo perioda, Moskva, Nauka, 1972, R 815

TIHOMIROV, Litovskaâ SSR : period 1966-1970 : vypusk 1, pečatnye raboty, Vilnius, Periodika, 1975, R 904

VOROB'EV, Vladimir Vasil'evi', Formirovanie naseleniâ Vostočnoĭ Sibiri : geograficeskie osobennosti i problemy, Novosibirsk, Izd-vo "Nauka," Sibirskoe otd-nie, 1975, R 908

VOSKRESENSKIJ, Geomorfologiâ Amuro-Zejskoj ravniny i nizkogor'â Malogo Hingana : čast' 2, Moskva, izd. Moskovskogo Universiteta, 1973, R 929 <2>

France 

G 10-5478 G 10-5425 G 10-5481

G 10-5472 2007, G 10-5432

ADOUMIÉ, Géographie de la France, Paris, Hachette Supérieur, DL 2007, G 10-5430

AGHION, ARTUS, COHEN, Mondialisation : les atouts de la France, Paris, la Documentation française, DL 2007, G 10-5433

ALBANEL, AUBRY, MAUROY, BECKARY, Pays'âges : ... au début, il y avait la forêt :

16 décembre 2007 - 03 août 2008, Lille, Musée d'Histoire naturelle de Lille, DL 2007,

ALBERTINI, Jean-Benoît, LE DOLLEY, ALOUIS, LESPIAUCQ, Les contrats de projets EtatsRégions, Paris, La Documentation française : DIACT, DL 2007, G 10-5471

ALLEMAND, Sylvain, Délocalisations : la catastrophe n'aura pas lieu, Paris, les Carnets de l'info-Scrineo, DL 2007, G 10-5449

ANGELIER, Jean-Pierre, CHEVALIER, Économie des industries de réseau, Grenoble, Presses universitaires de Grenoble, DL 2007, G 10-5462

ARCHAMBAULT, Paul, SEGALEN, Les enfants de familles désunies en France: leurs trajectoires, leur devenir, Paris, Institut national d'études démographiques, DL 2007,

BIROT, Pierre, Etude comparée de la vie rurale pyrénéenne dans les pays de Pallars (Espagne) et de Couserans (France) : thèse pour le doctorat ès-lettres présentée à la faculté des Lettres de l'Université de Paris, Paris, J.-B. Baillière, 1937, G 14-217

BODY-GENDROT, Sophie, Sortir des banlieues : pour en finir avec la tyrannie des territoires, Paris, Autrement, impr. 2007, G 10-5480

BOUVIER, Jean-Claude, Les noms de rues disent la ville, Paris, C. Bonneton, impr. 2007,

CARADEC, Vincent, Quand les retraités partent en vacances, Villeneuve d'Ascq, Presses universitaires du Septentrion, impr. 2007, G 10-5439

CAVAILLÈS, Henri, La route française, son histoire, sa fonction : étude de géographie humaine, Paris, Armand Colin, 1946, G 13-129

CHAPUIS, Olivier, Cartes des côtes de France : histoire de la cartographie marine et terrestre du littoral, Douarnenez, Editions du Chasse-Marée, impr. 2007, G 10-5438

CHAPUIS, Robert, MILLE, FRANCART, Vers des campagnes citadines, le Doubs (1975-2005), Besançon, Éditions Cêtre : Presses Universitaires de Franche-Comté, DL 2007,

Chartes d'urbanisme commercial : leur contenu, les premiers enseignements, Lyon, CERTU, 1999, G 10-5427

COTTE, Michel, Le choix de la révolution industrielle : les entreprises de Marc Seguin et de ses frères (1815-1835), Rennes, Presses universitaires de Rennes, 2007, G 10-5444

DELAPORTE, Pierre, Développement durable : 21 patrons s'engagent, Paris, le Cherche midi, DL 2002, G 10-5465

DELFOSSE, Claire, La France fromagère (1850-1990), [Paris], La Boutique de l'Histoire, impr.

DIKEÇ, Mustafa, Badlands of the Republic : space, politics and urban policy, Malden ; Oxford, Blackwell publ., cop. 2007, G 10-5429

DUBOIS, Jacques, LE MOUËL, Volcans actifs français et risques volcaniques : Martinique, Guadeloupe, Réunion, Pacifique, Paris, Dunod, impr. 2007, G 10-5442 

G 10-5464 2003, G 10-5466 G 10-5455 G 10-5463 2007, G 10-5443 2007, G 10-5426 G 10-5445 1967, G 10-1119 2007, G 10-5470

DUMONT, René, Voyages en France d'un agronome, Paris, Libr. de Médicis :

Ed. M.-Th. Génin, 1951, G 10-5475

Enjeux et défis de l'industrie en Île-de-France, Paris, DRIRE Île-de-France, DL 2007,

Étude prospective sur les lycées dans les Pays-de-la-Loire, Nantes, INSEE Pays-de-laLoire, 2007, G 10-5409

FOLLENFANT, Teddy, Développement durable : 21 maires s'engagent, Paris, le Cherche midi,

GRESLE-POULIGNY, Cartographier la Corse au temps de Pasquale de Paoli : les cartes et l'histoire, 1755-1807 : [exposition, Corte, Musée régional d'anthropologie de la Corse, 23 juin-29 décembre 2007], Corte ; Ajaccio, Musée de la Corse : Albiana, impr. 2007,

GUILLAUME, Jacques, La France dans l'Union Européenne, Paris, Belin, DL 2007,

HAAS, VIGNERON, Évaluation \& territoires, Paris, la Documentation française : [DIACT], DL 2007, G 10-5412

JACOMET, Dominique, CHEVALIER, Mode, textile et mondialisation, Paris, Economica, DL

JAUZE, SAFFACHE, Approche des littoraux réunionnais et martiniquais, Saint-Denis (Réunion), Université de la Réunion, Faculté des lettres et des sciences humaines, impr.

JEAN-BRUNHES DELAMARRE, Mariel, Le Berger dans la France des villages : Bergers communs à Saint-Véran en Queyras (Hautes-Alpes) et à Normée en Champagne, (Marne) : Une étude comparée d'ethnologie et de géographie humaine, Paris, Éditions du Centre National de la Recherche Scientifique, 1970, G 10-5473

La France et le monde : bilan d'une présidence 1995-2007, Paris, Géoéconomie, 2007,

LAFAY, Gérard, France horizon 2050: dynamique mondiale et défis français, Paris, Economica, DL 2007, G 10-5440

LE CARO, Yvon, Les Loisirs en espace agricole : L'expérience d'un espace partagé, Rennes, Presses Universitaires de Rennes, 2007, G 10-5434

LE LANNOU, Maurice, Le déménagement du territoire : rêveries d'un géographe, Paris, Seuil,

Les fonds structurels européens 2007-2013, Paris, la Documentation française : [DIACT], DL

LÉTANG, Pierre, Urbanisme commercial : réglementation du commerce de détail, équipements commerciaux, cinématographiques et hôteliers, créations, extensions, transferts, Paris, le Moniteur, 1997, G 10-5428

MABIALA-GASCHY, Christian Gilbert, La France et son immigration : tabous, mensonges, amalgames et enjeux, Paris, l'Harmattan, DL 2007, G 10-5453

MAILlARD, Jacques de, Vin et politique : Bordeaux, la France, la mondialisation, Paris, Presses de Sciences Po, impr. 2007, G 10-5456

MARTINETTI, Joseph, Géopolitique de la Corse, Paris, A. Colin, DL 2007, G 10-5435 

G 10-5479 G 10-5436 2006, G 9-6564 G 9-6531

MEYNIER, André, Histoire de la pensée géographique en France : 1872-1969, Paris, Presses universitaires de France, 1969, G 10-1320

MICHELET, Jules, CHEVRIER, Tableau de la France, Paris ; Niort, Ed. Nicolas, 1947,

MOLLARD, SAUBOUA, HIRCZAK, Territoires et enjeux du développement régional, Versailles, Quae, DL 2007, G 10-5451

Montpellier (Languedoc-Roussillon) L.R. . Technopole, [S.l.], [s.n.], [s.d.], G 10-2691-2

NÈGRE, Ernest, Les noms de lieux en France, Paris, A. Colin, impr. 1963, G 10-5474

Paris sous l'oeil des chercheurs, Paris, Belin, DL 2007, G 10-5460

PELISSON, Eric, Les discriminations, Paris, Ellipses, DL2007, G 10-5441

PITTE, Jean-Robert, La France, Paris, A. Colin, DL 2005, G 10-4996

Pour un redécoupage des régions françaises : mondialisation économique et taille des régions, Paris, l'Harmattan, DL 2007, G 10-5454

SUBRA, Philippe, Géopolitique de l'aménagement du territoire, Paris, A. Colin, DL 2007,

TSIOMIS, ZIEGLER, Anatomie de projets urbains : Bordeaux, Lyon, Rennes, Strasbourg, Paris, Éd. de La Villette, impr. 2007, G 10-5447

TUTENUIT, Claire, Développement durable, cinq ans après : la métamorphose, Paris, Le cherche midi, DL 2007, G 10-5467

Géographie Humaine

ALLEMAND, Sylvain, GODARD, Le développement durable, Paris, Editions Autrement, DL

AMANN, Jürgen M., Mythos Interkulturalität? : die besondere Problematik deutschsyrischer Unternehmenskooperationen, München ; Wien, Profil, 2007, G 9-6583

ARNAUD-BABOU, Isabelle, Les dilemmes du tourisme, Paris, Vuibert, DL 2007, G 9-6580

Atlaséco 2008 : atlas économique et politique mondial : 1000 tableaux pour tout comprendre, Paris, Le nouvel observateur, 2007, G 9-6607

AUBERTIN, ONG et biodiversité : représenter la nature ?, [Paris], IRD Editions, Institut de recherche pour le développement, 2005, G 9-6561

AUZELLE, Robert, Derniéres demeures : conception, composition, réalisation du cimetiére contemporain, Paris, 1965, G 9-1047

AZOULAY, Gérard, Les théories du développement : du rattrapage des retards à l'explosion des inégalités, Rennes, Presses universitaires de Rennes, 2002, G 9-6558

BADIE, Bertrand, L'état du monde : 2008, Paris, La découverte, 2007, G 9-6547

BAROU, Jacques, La planète des migrants : circulations migratoires et constitution de diasporas à l'aube du XXIe siècle, Grenoble, Presses universitaires de Grenoble, DL 2007,

BARRÉ, Bertrand, PIERRET, BAILLY, Atlas des énergies : quels choix pour quel développement ?, Paris, Éd. Autrement, impr. 2007, G 9-6544

BASTIÉ, Jean, La ville, Paris ; Milan ; Barcelone, Masson, 1991, G 9-3357

BATES, Marston, Les tropiques : l'homme et la nature entre le Cancer et le Capricorne, Paris, Payot, 1953, G 9-606 

2007, G 9-6563 1997, G 9-6627 2007, G 9-6608 2000, G 9-6560 G 9-6613 9-6597-<1> 2007, G 9-6568 2007, G 9-6576 2007, G 9-6570

BAUQUIS, Pierre-René, L'énergie d'aujourd'hui et de demain, Paris, Éd. Autrement, DL

BELL, David, Consuming geographies : we are where we eat, London ; New York, Routledge,

BELLEZZA, Giuliano, Geografia e beni culturali : riflessioni per una nuova cultura della geografia, Milano, F. Angeli, cop.1999, G 9-6610

BOBIN, Jean-Louis, L'énergie dans le monde : bilan et perspectives, Les Ulis, EDP Sciences, DL

BOURG, Dominique, Le développement durable : maintenant ou jamais, [Paris], Gallimard, DL 2006, G 9-6569

BOURGUIGNON, COHEN, LIPIETZ, Développement, Paris, La Documentation française,

CAPRON, HASCHAR-NOÉ, L'espace public urbain : de l'objet au processus de construction, Toulouse, Presses universitaires du Mirail, DL 2007, G 9-6548

CARRIER, LAURENT, Le phénomène urbain, [Paris], Aubier-Montaigne, DL 1965,

CHARVET, Jean-Paul, L'agriculture mondialisée, Paris, la Documentation française, 2007, G

CIATTONI, VEYRET-MEKDJIAN, Géographie et géopolitique des énergies, Paris, Hatier, DL

CIATTONI, VEYRET-MEKDJIAN, Les fondamentaux de la géographie, Paris, A. Colin, impr.

CLAUDE, Gérard, La Méditerranée : géopolitique et relations internationales, Paris, Ellipses, DL 2007, G 9-6571

CLAVAL, Paul, Régions, nations, grands espaces : géographie générale des ensembles territoriaux, Paris, M.-Th. Génin, 1968, G 9-1215

CLUZET, Alain, Ville libérale, ville durable ? : répondre à l'urgence environnementale, La Tourd'Aigues (Vaucluse), Éd. de l'Aube, DL 2007, G 9-6584

COMÉLIAU, Christian, La croissance ou le progrès ? : croissance, décroissance, développement durable, Paris, Éditions du Seuil, impr. 2006, G 9-6566

COOPER, DONAGHY, HEWINGS, Globalization and regional economic modeling, Berlin, Springer, cop. 2007, G 9-6552

COQUERY-VIDROVITCH, HÉMERY, PIEL, Pour une histoire du développement : États, sociétés, développement, Paris, l'Harmattan, DL 2007, G 9-6590

CSH occasional paper : [publication of the French research institutes in India], New Delhi, Centre de sciences humaines, 2001-, P 4600

DALBY, Ó TUATHAIL, Rethinking geopolitics, London, Routledge, cop. 1998, G 9-6626

DEFFONTAINES, Pierre, L'homme et la forêt, Paris, Gallimard, 1969, G 9-1437

DERCOURT, TISOT, Les géosciences au service de l'Homme : comprendre l'avenir, Strasbourg, Éditions Hirlé, cop. 2007, G 9-6591

DESHAIES, Michel, Les territoires miniers : exploitation et reconquête, Paris, Ellipses, DL 
DOLLFUS, Olivier, LÉVY, La mondialisation, [Paris], Presses de Sciences Po, impr. 2007, G 9-6575

DORTIER, VEGA, Dix questions sur la mondialisation, Auxerre, Sciences humaines, 2007, G 9-6594

DUCROUX, Les nouveaux utopistes du développement durable, Paris, Éd. Autrement, DL 2002, G 9-6562

DUPUY, Gabriel, La dépendance automobile : symptômes, analyses, diagnostic, traitements, Paris, Anthropos : diff. Économica, 1999, G 9-4632

DUPUY, GÉNEAU DE LAMARLIÈRE, Nouvelles échelles des firmes et des réseaux : un défi pour l'aménagement, Paris, L'Harmattan, DL 2007, G 9-6593

E \& H : économie \& humanisme, Caluire, Centre Économie et humanisme, 1964-1990, P 1118

ERKMAN, Suren, Vers une écologie industrielle, Paris, Ed. Charles Léopold Mayer, impr. 2004, G 9-6573

ÉTIENNE, Gilbert, Le développement à contre-courant, [Paris], Presses de Sciences Po, impr. 2003, G 9-6572

Explosion urbaine et mondialisation : points de vue du Sud, Louvain-la-Neuve ; Paris, Centre tricontinental : Éd. Syllepse, DL 2007, G 9-6603

EYDOUX, Henri-Paul, L'Homme et le Sahara, (Paris), Gallimard, 1943, G 9-129

FLEURET, THOUEZ, Géographie de la santé : un panorama, Paris, Economica : Anthropos, DL 2007, G 9-6599

FOUQUIN, SIROËN, Régionalisme et multilatéralisme sont-ils antinomiques ?, Paris, la Documentation française, 1998, G 9-4574

Globalisierung in der Zuckerdose : eine interaktive DVD-ROM des BMZ, Bonn ; Berlin, BMZ : DOKUFAKTUR, 2007, CD 306

GOLD, GOLD, Olympic cities : city agendas, planning and the world's games, 1896-2012, London ; New York, Routledge, cop. 2007, G 9-6551

GOTTMANN, Jean, La politique des États et leur géographie, Paris, A. Colin, 1952, G 9-584

GUILLOU, TAPIA, WADBLED, Migrations turques dans un monde globalisé : le poids du local, Rennes, Presses universitaires de Rennes, 2007, G 9-6581

HAGGETT, Peter, FRÉCHOU, L'analyse spatiale en géographie humaine, Paris, A. Colin, impr. 1973, G 9-1623

HALL, DU GAY, Questions of cultural identity, London ; Thousand Oaks (Calif.), Sage, cop. 1996, G 9-6624

HALL, Peter, GARA, Les villes mondiales, Paris, Hachette, DL1965, G 9-1447

HÉRUBEL, Marcel, L'homme et la côte : [étude d'économie maritime], Abbeville ; Paris, impr. F. Paillart : Gallimard, 1937, G 9-362

Ice, Cambridge, International Glaciological Society, 1958-, P 1334

JACKSON, Peter, Maps of meaning : an introduction to cultural geography, London ; New York, Routledge, 2001, G 9-6625

JEAN, Géographies de l'école rurale : acteurs, réseaux, territoires, Paris, Ophrys Géographie, cop. 2007, G 9-6605 

G 9-385 4643 4441

JUILLARD, Étienne, FLATRÈS, BLANC, Structures agraires et paysages ruraux : un quart de siècle de recherches françaises, Nancy, Impr. Berger-Levrault, 1957, G 9-884

KAHN, Régulation temporelle et territoires urbains : habiter l'espace et le temps d'une ville, Paris, L'Harmattan, DL 2007, G 9-6549

KAUL, GRUNBERG, STERN, Les biens publics mondiaux : la coopération internationale au XXIe siècle, Paris, Economica, 2002, G 9-6557

KAYSER, Bernard, L'agriculture et la société rurale des régions tropicales, Paris, Société d'édition d'enseignement supérieur, 1969, G 9-1291

KAZAZIAN, Il y aura l'âge des choses légères : design et développement durable, Paris, Victoires-Editions, 2003, G 9-6628

KOHL, Johann Georg, Der Verkehr und die Ansiedelungen der Menschen in ihrer Abhängigkeit von der Gestaltung der Erdoberfläche, Dresden und Leipzig, Arnold, 1841,

La Lettre du développement local, Paris, Entreprises territoires et développement, 1998-, $\mathbf{P}$

LAZAREV, Grigori, KALBERMATTEN, MICHEL, Vers un éco-développement participatif : leçons et synthèse d'une étude thématique, Paris, Éd. l'Harmattan, 1993, G 9-6559

LECAILLON, Jacques, Faut-il stopper la croissance?, Paris, Salvator, DL 2007, G 9-6546

LECERF, Maurice, Le fer dans le monde, Paris, Payot, 1942, G 9-410

LEGAULT, Albert, GRÉGOIRE-BLAIS, DELALANDRE, CHALMIN, Pétrole, gaz et les autres énergies : le petit traité, Paris, Éditions Technip, 2007, G 9-6588

LYNCH, Kevin, VÉNARD, VÉNARD, L'image de la cité, Paris, Dunod, 1969, G 9-1520

MASBOUNGI, GRAVELAINE, Penser la ville par la lumière, Paris, Éditions de la Villette, impr. 2003, G 9-5640

Moravian Geographical Reports, Brno, Institute of Geonics, Czech Academy of Sciences, $\mathbf{P}$

ORIGET DU CLUZEAU, Claude, Le tourisme culturel, Paris, Presses universitaires de France, impr. 2007, K 1054

PASTRÉ, La guerre mondiale des banques, Paris, PUF : Descartes \& Cie, DL 2007, G 9-6589

PAULET, Jean-Pierre, Les villes et la mer, Paris, Ellipses, DL 2007, G 9-6612

PLANHOL, Xavier de, Le monde islamique : essai de géographie religieuse, Paris, Presses universitaires de France, 1957, G 9-667

RALLET, TORRE, _La proximité à l'épreuve des technologies de communication _, Paris, L'Harmattan, 2007, G 9-6622

RATZEL, Friedrich, Anthropo-Geographie oder Grundzünge der Anwendung der Erdkunde auf die Geschichte, Stuttgart, J. Engelhorn, 1882, G 9-8

RATZEL, Friedrich, Anthropogeographie..., Stuttgart, J. Engelhorn, 1891, G 9-9

RAUFER, Atlas de l'islam radical, Paris, CNRS éditions, impr. 2007, G 9-6587

Religions \& territoires : quelle gestion locale des cultes ?, Paris, Pouvoirs locaux : diff. La documentation française, 2006, G 9-6574

SCARWELL, WEILL, Biocarburants, les temps changent ! : effet d'annonce ou réelle avancée ?, Villeneuve-d'Ascq, Presses universitaires du Septentrion, impr. 2007, G 9-6582 
SCHLAFFKE, Marlen, Von Bollenhüten und Ritterburgen : Tourismuswerbung und Raumbilder, München, Profil, cop. 2007, G 9-6578

SCHMITTHENNER, Henri, Les espaces vitaux et le conflit des civilisations, Paris, Payot, 1953, G 9-607

SMOUTS, Le développement durable : les termes du débat, Paris, A. Colin, 2005, G 9-6567

SORRE, Maximilien, Les migrations des peuples : essai sur la mobilité géographique, Paris, Flammarion, cop. 1955, G 9-628 BIS

TERNY, D'où vient la richesse des nations?, Paris, Economica : Banque internationale pour la reconstruction et le développement, DL 2007, G 9-6598

TSAFACK NANFOSSO, L'économie solidaire dans les pays en développement, Paris, 2007, G 9-6611

Unasylva, 1947-2006 : an international journal of forestry and forest industries, Rome, Food and agriculture organization of the United Nations, cop. 2007, CD 303

VALANTIN, Jean-Michel, Ecologie et gouvernance mondiale, Paris, Autrement, impr. 2007, G 9-6565

VANOLO, Alberto, Gli spazi economici della globalizzazione : geografie del commercio internazionale, Torino, UTET Università, cop. 2007, G 9-6609

VERDAVOINE-BOURGET, Francis, Surpeuplement : un drame planétaire !, Coulommiers, Dualpha éd., cop. 2007, G 9-6579

VEYRET-MEKDJIAN, Dictionnaire de l'environnement, Paris, A. Colin, DL 2007, W 357

VEYRET-MEKDJIAN, Le développement durable, Paris, Éd. Sedes, impr. 2007, G 9-6602

WIEL, Marc, Pour planifier les villes autrement, Paris, l'Harmattan, DL 2007, G 9-6550

Géographie Physique

ARCHAMBAULT, Michel, Documents et méthode pour le commentaire de cartes : géographie et géologie : avec 44 figures dans le texte, 6 planches et 15 cartes en couleurs hors texte, Paris, Masson et Cie, 1967, G 8-1340-<2>

ARNOULD, Paul, Géographie de l'environnement, Paris, Belin, DL 2007, G 8-3190

BALLAND, Les eaux cachées : études géographiques sur les galeries drainantes souterraines, Paris, Université de Paris-Sorbonne, 1992, G 8-2378

BOUGLET, Brice, LE COEUR, L'efficacité de l'érosion glaciaire dans les montagnes de l'Andorre, [S.l.], [s.n.], 2005, CD 309

BOULVERT, Yves, Carte morphopédologique interactive de la République de Guinée à 1:200 000, Paris, IRD, cop. 2005, CD 308

BRUNET, Fabrice, La Terre interne : roches et matériaux en conditions extrêmes, Paris, Vuibert : Société géologique de France, DL 2007, G 8-3188

LE ROY LADURIE, Emmanuel, VASAK, Abrégé d'histoire du climat du Moyen Âge à nos jours, Paris, Fayard, impr. 2007, G 8-3189

2 PARCEVAUX, Sané de, Bioclimatologie : concepts et applications, Versailles, Éditions Quae, 2007, G 8-3187

PIRAZZOLI, Paolo Antonio, Les littoraux : leur évolution, [Paris], Nathan, cop. 1993,

G 8-2301 

1972, K 695

Mélanges

Périodiques P 4458-32/2007 4603 1929-, P 835 P 797-5260

ROUBAULT, Marcel, La dérive des continents, Paris, Presses Universitaires de France,

VENNIN, Facies from palaeozoic reefs and bioaccumulations, Paris, Publications scientifiques du Muséum, 2007, G 8-3193

VISCONTI, Guido, Clima estremo : un'introduzione al tempo che ci aspetta, Milano, Boroli Ed., cop. 2005, G 8-3192

ALLIX, André, Propos d'un géographe, [Lyon], Revue de géographie de Lyon, 1960, G 6-86

BLACHE, Jules, Pages géographiques, Gap, Editions Ophrys, 1963, G 6-103

LANDY, LÉZY, MOREAU, Les raisons de la géographie : itinéraires au Sud avec JeanPierre Raison, Paris, Karthala, impr. 2007, G 6-331

Monde arabe -Proche-Orient

ABABSA, Myriam, CALCAVECHIA, Amman : de pierre et de paix, Paris, Éd. Autrement, impr. 2007, G 24-388

CHERKAOUI, Mohamed, Le Sahara : liens sociaux et enjeux géostratégiques, Oxford, The Bardwell press, 2007, G 24-394

HEJAZEEN, Emad Galanzeh, Tourism and local communities in Jordan : perception, attitude and impacts, München; Wien, Profil, cop. 2007, G 24-389

Ouvrages de référence

LORIMER, WITHERS, Geographers : biobibliographical studies, London ; New York, Continuum, cop. 2007, A 5.1 31-<26>

NIDIAU, Fabrice, L'anglais du tourisme : [étudiants, stagiaires et professionnels], LevalloisPerret, Studyrama, impr. 2007, W 358

Acta geographica, Paris, Société de géographie, 1947-2000, P 976\%70

Actes du colloque «L'Europe rhénane et l'Europe centrale - dynamique et mutations »: colloque international, [Metz] 11-13 mars 1998 : hommage au Professeur François Reitel, Metz, Presses universitaires de Metz, 1999, P 3284-24-1/2

Ajia Taiheiy? kenky?, Musashino, Tokyo, Seikei Daigaku. Ajia Taiheiy? Kenky? Sent?,

Allemagne-Est : séquelles du passé et nouvelle architecture des relations, Paris, la Documentation française, DL 2005, P 3379-1049

Ambiente società territorio. Geografia nelle suole : rivista dell'Associazione italiana insegnanti di geografia, Roma, Associazione italiana insegnanti di geografia, 2001-, P

Anais da Academia Brasileira de Ciências, Rio de Janeiro, Academia Brasileira de Ciências,

ANDOLFATTO, Les syndicats en France, Paris, la Documentation française, DL 2007,

Annales de géographie, Paris, A. Colin, 1891-, P 523\%1941

Annuaire suisse de politique de développement, Genève, Institut universitaire d'études du développement, cop. 2003-, P 4731-26/1

Annual review of environment and resources, Palo Alto, Annual Reviews, 2003-, P 3807-32 

P 3379-1057 P 1652/A 3961 P 3379-1039 4480 P 1686 P 3367-943 P 3633 4404-151

Antipode : a radical journal of geography, Worcester, Mass. ; Worcester, Mass. ; Oxford, Antipode : Antipode : Blackwell, 1969-, P 3573

Arctic, Calgary, Artic Institute of North America, 1948-, P 973

Asia Pacific journal of tourism research, Pusan ; Houston, Tex, Asia Pacific Tourism Association : University of Houston, Conrad N. Hilton College, P 4693-12/4

Asie centrale : un enjeu géostrategique, Paris, La Documentation française, DL 2006,

Australian Geographer, Sydney, Geographical Society of New South Wales, 1928-, P 280

Autrepart : Revue de sciences sociales au Sud, La Tour d'Aigues ; Bondy ; La Tour d'Aigues ; Bondy ; Paris, Éd. de l'Aube : ORSTOM : Éd. de l'Aube : ORSTOM : A. Colin, 1997-,

Aval : lettre statistique et économique de Haute-Normandie, Rouen, INSEE, 1980-, P

Balkans : vers un nouvel espace de coopération, Paris, la Documentation française, 2003,

BIBLIOthèque(s) : revue de l'Association des bibliothécaires français, Paris, Association des bibliothécaires français, 2002-, P 2234

Bollettino della Società geografica italiana, Roma, Società geografica italiana, 1944-, P 164

BOYLE, FINDLAY, Population, space and place, Chichester, U.K., J. Wiley \& Sons, 2004-, P

BRÉCHON, Les élections présidentielles en France : quarante ans d'histoire politique, Paris, la Documentation française, DL 2007, cop. 2008, P 797-5266/67

Caribbean journal of science, Mayagüez, Puerto Rico, University of Puerto Rico, 1961-,

CARIO, Les droits des victimes d'infraction, [Paris], [la Documentation française], DL 2007,

Cartographic journal, London, British Cartographic Society, 1964-, P 4063

Catena, Cremlingen ; Cremlingen ; Giessen ; Amsterdam, Catena-Verlag : Catena-Verlag : Lenz : Elsevier Science, 1973-, P 3490

CHARDON BADERTSCHER, Perspectives urbaines, Neuchâtel, Société neuchâteloise de géographie, cop. 2006, P 68-49

CHARILLON, ROUGIER, Afrique du Nord, Moyen-Orient : Moyen-Orient : la triple impasse, Paris, la Documentation française, DL 2007, P 797-5262/63

CHURCHILL, Population and development review, New York, Population Council, 1975-,

Ciudad y territorio, estudios territoriales, Madrid, Ministerio de Fomento, 1993-, P

CLARKE, SMELLIE, Papers from the International Symposium on Earth and Planetary Ice-Volcano Interactions held in Reykjavík, Iceland, on 19-23 June, 2006, Cambridge, International Glaciological Society, c2007, P 3731-45

CLAVAL, Géographie historique des villes d'Europe occidentale : actes, Paris, Département de géographie, 1984-1986, P 3905-<4> 
Commodity chains : Special issue, Berlin, Gesellschaft für Erdkunde zu Berlin, 2007,

P 6-138-2

D’AGOSTINO, Meridione : Sud e Nord nel mondo : rivista bimestrale, Napoli, Edizioni Scientifiche Italiane, 2001-, P 4604-3

DALIGAUX, DURBIANO, Les grands sites naturels des aires métropolitaines méditerranéennes : Hommage au professeur Roland Courtot, Aix-en-Provence, Institut de géographie, 2005, P 1420-105

DARBOT-TRUPIANO, Stéphanie, PITTE, Le partenariat euro-méditerranéen : une géographie politique des relations nord-sud : thèse en vue d'obtenir le grade de docteur de l'Université de ParisIV-Sorbonne, [S.1.], [s.n.], 2007, T 2026

DARQUES, Transitions balkaniques, Aix-en-Provence, Association des « Amis de la Revue Méditerranée » : Institut de géographie, 2004, P 1420-103

DAVIET, MIOCHE, WEBER, Entreprises en Méditerranée : héritages, modèles, redéploiements, Aix-en-Provence, Institut de géographie, 2006, P 1420-106

DESVIGNES, Stratégies de petits prix, Paris, Éd. touristiques européennes, 2003, P 4178-79

Développement durable, Paris, A. Colin, 2007, P 497/A-71-3

Dili Kexue, Beijing, Kexue Chubanshe, P 3847

Dossier « Tourisme et solidarité », Montréal, Université du Québec, 2007, P 4703-26-3

DURBIANO, BARTHES, Nouvelles dynamiques du développement rural dans les Alpes du Sud, Aix-en-Provence, Institut de géographie, 2006, P 1420-107

Earth-science reviews, Oxford ; Lausanne ; New York ; Shannon ; London ; Amsterdam, Elsevier, P 1825

East Asian cross-regionalism, Vancouver, B.C., University of British Columbia, c2007, P 1623-80-2

Economie et statistique, Paris, INSEE, 1969-, P 2292

Économie rurale : bulletin de la Société française d'économie rurale, Paris, SFER, 1953-, P 1129-297/298

Effets de la pollution atmosphérique sur les matériaux, Paris, 2007, P 4705/A , octobre 2007

Environment \& planning, London, Pion, 1974-, P 3542

Environment and behavior, [Beverly Hills, Calif.], Sage Publications, 1969-, P 3282

Environmental conservation, Lausanne, Elsevier Science : Elsevier Sequoia, P 3493

Espaces naturels : revue des professionnels des espaces naturels, Bastia, Mediaterra, 2003-,

P 4662

Espaces, Paris ; Paris, Association pour la culture par les loisirs et le tourisme : Editions touristiques européennes, 1970-, P 3270

Etudes caribéennes, Paris, Ed. Publibook, 2004-, P 4679-6

Europe centrale et orientale 2003-2004 : retrouvailles européennes, Paris, la Documentation française, DL 2004, P 3379-1044

Europe centrale et orientale 2004-2005, Paris, La Documentation française, 2005, P 3379-1050 
Europe centrale et orientale 2005-2006, Paris, La Documentation française, DL 2006, P 3379-1056

Europe centrale et orientale 2006-2007, [Paris], [la Documentation française], DL 2007, P 3379-1062

European urban and regional studies, London, Sage, 1994-, P 4422

Eye on Alaska : Special issue, London, Taylor \& Francis, 2007, P 3613-30-3/4

FAUST, Heiko, Vergleichende Kulturgeographie : empirische Befunde regionaler Integrationsprozesse in tropischen Agrarkolonisationsräumen Boliviens, der Elfenbeinküste und Indonesiens, Göttingen, Niedersachs, Goltze, 2007, P 1004-116

Fennia : Bulletin de la société de géographie de Finlande, Helsinki, helsingfors, 1889-, P 89-185/1

Feuillets d'informations : publication à caractère méthodologique de la Fédération des Professeurs de Géographie, Liège, Fédération des Professeurs de Géographie, P 4473

FLITNER, Michael, Lärm an der Grenze : Flugärm und Umweltgerechtigkeit am Beispiel des binationalen Flughafens Basel-Mulhouse, Wiesbaden, F. Steiner, 2007, P 3 BIS-140

FOURNIER, Géographie des idées : les archipels du savoir, P 4384-189

G.E.O. : écologie, environnement, organisation de l'espace, Liège, Fédération des Professeurs de Géographie, P 1704

Géochronique, Paris, Bureau de recherches géologiques et minières : Société géologique de France, 1982-, P 441

Géoéconomie, Paris, Géoéconomie, 2000-, P 4524

Geografický ?asopis, Bratislava, SAP-Slovak Academic Press s.r.o, P 1236-59/1

Geografie, Utrecht, Koninklijk Nederlands Aardrijkskundig Genootschap, P 4352

Geographical Review of Japan : English Edition, T?ky?, Nihon chiri gakkai, P 1063

Géographie et cultures, Paris, L'Harmattan, 1992-, P 4350-32

Geographische Rundschau, Braunschweig, G. Westermann, 1949-, P 1217

GeoJournal, Dordrecht ; London ; Boston, Kluwer Academic Publishers, 1977-, P 3794

Geologica acta, Barcelona, Facultad de Geologia, Universidad de Barcelona, 2003-, P 4646

Geological Society of America bulletin, [New York, N.Y.], The Society, [1961-,

P 697-120-1/2

Géomorphologie : relief, processus, environnement, Paris, Sedes, 1995-, P 4494

Geomorphology and ecosystems : $336^{\text {th }}$ Binghamton Geomorphology Symposium_, Amsterdam ; London ; New York, Elsevier, 2007, P 4168-89-1/2

Geomorphology, Tokyo ; Oxford ; New York ; Amsterdam, Elsevier, 1987-, P 4168

Géostratégiques, Paris, IIES, 2001-, P 4610-18

GODARD, Miradas cruzadas sobre la influencia intelectual, cultural y científica entre Perú y Francia, Lima, Institut Français d'Études Andines, 2007, P 3386-36-1

GRAVEL, Nicolas, Is India better off today than 15 years ago ? : a robust multidimensional answer, New Delhi, Centre de sciences humaines, cop. 2007, P 4600-20

GREFFE, Xavier, Artistes et marchés, Paris, la Documentation française, DL 2007,

P 797-5264/65 
Growth and change, [Lexington, University of Kentucky, College of Business and Economics], P 3539

GUINEA BUENO, BOUCHARD, Avances de investigación en el Ecuador prehispánico, Lima, Institut Français d'Études Andines, 2006, P 3386-35-3

HÄGERSTRAND, BUTTIMER, Geographers of Norden : reflections on career experiences, Lund, Sweden, Royal University of Lund, Dept. of Geography, Lund University Press, 1988, P 1100/B-52

Hallesches Jahrbuch für Geowissenschaften, Halle, Saale, Institut für Geologische Wissenschaften und Geiseltalmuseum der Martin-Luther-Universität Halle-Wittenberg, P 3654/B-23

Health and place, Oxford, Pergamon, 1995-..., P 4454

HEINRITZ,_Geographie in München _ :_disziplingeschichtliche Streifzüge _, München, Universität München, 2007, P 18-89

HENRY, Laurence, Trade and economic arrangements between India and South East Asia in the context of regional construction and globalisation, New Delhi, Centre de Sciences Humaines, 2007, P 4600-19

HERGET, The fluvial system : past and present dynamics and controls, Amsterdam ; Boston ; Jena, Elsevier, 2007, P 4168-92-3/4

Hérodote : revue de géographie et de géopolitique, Paris, Maspero, 1976-, P 3536

HIRSCHHAUSEN, LACQUEMENT, Le développement rural en Allemagne réunifiée, P 3740-38-3

Holocene, Sevenoaks, Edward Arnold, P 4415

îles : ces étranges objets de désir, Grenoble, Glénat, 2008, P 976/A-1

In memoriam Helmer Smeds : 29.7.1908-8.12.1967, Helsinki, Societas geographica Fenniae, 1968, P 90-20

Insula, Paris, International Scientific Council for Island Development, P 4414-16/1

International journal of urban and regional research, London, Edward Arnold (Publishers) Ltd, 1977-, P 3611

International planning studies, Abingdon, Carfax, P 4525

Investigaciones geográficas - Instituto de Geografía. Universidad Nacional Autónoma de México, México, DF, Instituto de Geografía. UNAM, P 3153-62

Investir au Mali, Paris, Promoguide, 2007, P 1101/A-1

Itinéraires culturels à l'Est : l'empreinte du politique, Paris, la Documentation française, DL 2006, P 3379-1058

J'ai 40 ans..., Paris, la Documentation française, 2004, P 3379-1046

JOLY, Arménie : reconstruction et avenir d'un pays du Sud Caucase, Paris, Société de Géographie, 2007, P 976/A-HS-1527-2

JOLY, Jean-Baptiste Charcot (1867-1936), Paris, Société de Géographie, 2007, P 976/AHS-1527-1

JOSSA, Edward, Les finances des départements 2005, Paris, Direction générale des collectivités locales, impr. 2007, P 4349 (2005)

Journal of contemporary European studies, Abingdon, Corfax, P 4394/B-15/1 
Journal of European area studies, Abingdon, Carfax, P 4394/A

Journal of geography, Indiana, PA, National Council for Geographic Education, 1902-, P 247

Journal of rural studies, Oxford ; New York, Pergamon, 1985-..., P 4212

Journal of transport geography, Oxford, Butterworth-Heinemann, 1993-..., P 4379

JOUSSEAUME, Valérie, Patrimoine, culture et construction identitaire dans les territoires ruraux, Rennes, Presses universitaires de Rennes, DL 2007, P 1167-204-3

JQS. Journal of quaternary science, Harlow, Essex, Longman, 1986-, P 4222

KOBER, GLÉNAT, La GéoGraphie : Terre des hommes, Grenoble, Glénat, 2008-, P 976/B-1

KOFFI, Marthe Adjoba, CHALÉARD, Mutations sociales et gestion de l'espace rural en pays ébrié (sud-est de la Côte d'Ivoire) : thèse pour l'obtention du doctorat en géographie, [s.l.], [s.n.], 2007, T 2027

Kölner Geographische Arbeiten, Koln, Geographisches Institut der Universitat Koln, 1952-, P 1153-87

L' Investissement en URSS : choix et financement, Paris, La documentation française. Centre français du commerce extérieur, 1976, P 3379-202

L'espace baltique : isthme russo-européen?, Paris, la Documentation française, DL 2005, P 3379-1048

L'Information agricole, Paris, Confédération générale de l'agriculture, 1952-, P 1886

L'Information géographique, Paris, Baillière, 1936-, P 497

La Cité Nationale de l'Histoire de l'Immigration : Quels publics ?, Paris, Hommes \& migrations, 2007, P 4530/A\%07 hors-série

La crise iranienne: mythe ou réalité ?, Paris, Institut international d'études stratégiques, 2008, P 4610-18

La mondialisation jusqu'aux marges du monde : la pluie ou le beau temps?, Pessac, Presses universitaires de Bordeaux, DL 2007, P 945-60/238

La Recherche, Paris, Société d'éditions scientifiques, 1970-, P 4551

La Russie et les autres pays de la CEI 2001-2002 : Ukraine, Biélorussie, Moldavie, SudCaucase, Asie centrale, Paris, la Documentation française, 2002, P 3379-1030

50 La Russie et les autres pays de la CEI en 2004, Paris, la Documentation française, DL 2005, P 3379-1047

La Russie et son étranger proche : de l'usage du soft power, Paris, la Documentation française, DL 2006, P 3379-1055

La symétrie et ses doubles: approches géographiques, Nancy, Association de Géographes de l'Est : Université de Nancy 2, 2007, P 1422-47-2

3 LANG, GLADE,_Challenges in geomorphological methods and techniques _ : special issue, Amsterdam ; Boston ; Jena, Elsevier, 2008, P 4168-93-1/2

Le bilan de l'économie française 2006/2007, Paris, la Documentation française, 2007,

P 1102-2932

55 Le changement climatique, un défi mondial, Paris, la Documentation française, 2007, P 1102-2930 
Le Courrier des pays de l'Est : mensuel d'informations économiques, Paris, la Documentation française, 1964-, P 3379

Le Courrier, Paris, ANDAFAR, 1997-2004, P 4709

Le Journal de la marine marchande, Rueil-Malmaison, Groupe Liaisons, 2001-, P 1245

Le Monde des cartes, Paris, Comité français de cartographie, 2002-, P 1773

Le risque : de la recherche à la gestion territorialisée, Lyon, Revue de géographie de Lyon, 2007, P 487-82

Le Sud-Caucase : recomposition régionale et nouvelles alliances, Paris, La Documentation française, 2004, P 3379-1043

LEENHARDT-SALVAN, Sites religieux \& tourisme, Paris, Éd. touristiques européennes, DL 2007, P 4198-96

Les Cahiers d'Outre-mer : revue de géographie de Bordeaux et de l'Atlantique, Bordeaux, Institut de la France d'Outre-mer, 1948-, P 945

Les Cahiers scientifiques du transport, Caen ; Lyon, Paradigme : Association française des instituts de transports, 1994-, P 3855

Les États d'Asie centrale face à l'indépendance : Ouzbékistan, République kirghise, Tadjikistan, Turkménistan, Paris, la Documentation française, 1994, P 3379-388

Les mondes patronaux, Paris, Presses de Sciences Po, DL 2007, P 4724-68

Les nouveaux voisins orientaux de l'Europe élargie, Paris, La Documentation française, 2004, P 3379-1042

Les pays de la CEI 2000-2001 : le 11 septembre, une date charnière, Paris, la Documentation française, 2001, P 3379-1020

Les politiques d'immigration à l'Est, Paris, la Documentation française, DL 2007, P 3379-1060

Letopis' Severa, Moskva, izd-vo. “MYSL', 1975, R 954

MADAVAN, Delon, Jaffna et le conflit intercommunautaire à Sri Lanka, Paris, Pôle de recherche pour l'organisation et la diffusion de l'information géographique, DL 2007,

P 4569-32

MAKKI, EIDAM, Böden im städtischen Umfeld, Berlin, Geographisches Institut der Humboldt-Universität, 2007, P 1288-108

Man \& development, Chandigarh, Centre for Research in Rural \& Industrial Development, 1979-, P 4158

Man \& environment, Pune, Indian Society for Prehistoric and Quaternary Studies, 1977-, P 3603-32/

75 MARÉCHAL, Jean-Paul, Développement durable, les vrais enjeux : dossier spécial, Paris, Choiseul, 2007, P 4524-44

Mediterranean landscapes, Berlin, Gesellschaft für Erdkunde zu Berlin, 2007, P 6-138-1

Météo hebdo, Boulogne-Billancourt, Direction de la météorologie nationale, 1986-, P 2186

Meteorologiâ i gidrologiâ, Moskva, Gidrometeoizdat, 1935-, P 1684-12

Meteorological applications, Reading, Royal Meteorological Society, P 4457

Météorologie, Paris, Société météorologique de France, 1925-, P 512 
Minorités à l'Est : variations sur la reconnaissance identitaire, Paris, la Documentation française, DL 2005, P 3379-1052

MIRAMONT, Géosystèmes montagnards et méditerranéens : un mélange offert à Maurice Jorda : miscellany presented to Maurice Jorda, Aix-en-Provence, Association des «Amis de la Revue Méditerranée » : Institut de géographie, 2004, P 1420-102

Mondialisation, Paris, A. Colin, 2007, P 497/A-71-2

Montagnes espagnoles, Toulouse, Presses universitaires du Mirail, 1990, P 460-61-<2>

Monthly weather review, [Washington, D.C.], War Dept., office of the Chief Signal officer, P 2034

MORHANGE, GOIRAN, MARRINER, Environnements littoraux méditerranéens, héritages et mobilité, Aix-en-Provence, Institut de géographie, 2005, P 1420-104

Mountain research and development, Boulder, Colo ; Tokyo, International Mountain Society : United Nations University, 1881-, P 4167

MÜLLER, KNAPPE, Ländliche Gesellschaft europäischer Peripherien, Leipzig, Leibniz-Institut für Länderkunde, cop. 2007, P 4694-7

National geographic, Paris, National geographic France, 1999-, P 250

Niveaux de vie à l'Est : inégalités et pauvreté, Paris, la Documentation française, DL 2005,

P 3379-1051

Note de conjoncture de l'INSEE, Paris, Institut national de la statistique et des études économiques, 1985-, P 4751-mars

NPI : navigation ports \& industries, Strasbourg, Ed. de la navigation du Rhin, 2004-, P 2112

Objectif Terre 2050, Paris, Société d'Éditions Scientifiques, 2008, P 4551/B-415 (cahier spécial)

OFFNER, Jean-Marc, Le Grand Paris, Paris, la Documentation française, DL 2007, P 3367-942

Östliches Hochasien : Grenzraum zwischen Indien und China ; ; Sikkim, Indiens Tor zu Tibet; Wandel bei den Nomaden in Osttibet ; Von Natur aus? Osttibets Hochweiden ; Nachhaltige Bodennutzung in Bhutan ; Forum: Wasserkraft aus Yunnan ; Globalisierung und Weltmarkt für Zucker, Braunschweig, Westermann, 2007, P 1271\%07-11

PAUL, Frank, The new Swiss glacier inventory 2000 : application of remote sensing and GIS, Zürich, Geographisches Institut der Universität, cop. 2006, P 3881-52

Paysages alpins en perspective: Views and prospects, Grenoble ; Paris, Revue de géographie alpine : A. Colin, 2007, P 505-95-4

Penn ar bed, Brest, Société pour l'étude et la protection de la nature en Bretagne, 1953-, P1162-201

PENNING-ROWSELL, Environmental hazards : human and policy dimensions, Amsterdam, Elsevier, 2007-, P 4586-7/3

Permafrost and periglacial processes, Chichester, Sussex, Wiley, 1990-, P 4450

Perspektiven, Wien, Compress Verlagsges.m.b.H, P 3461

PETRESCU, Studia Universitatis Babes-Bolyai, Cluj-Napoca, Universitatea "Babe?-Bolyai, 2007-, P 1381/A-1-1/2 
Photo interprétation: images et représentations spatiales de l'environnement, Paris ; Paris ; Paris, Editions technip : Editions technip : Éd. Eska, 1962-, P 1665-43/1

Physical geography, Silver Spring, MD, V.H. Winston, P 3939

Polar geography, Palm Beach, FL ; New York ; London, V.H. Winston and Son : Distributed by Bellwether Pub : Taylor \& Francis, c1995-, P 3613-30-1/2

Polar Record, Cambridge, Scott Polar Research Institute, 1931-, P 1047-44/228

Political geography, Oxford, Butterworth-Heinemann, 1992-..., P 3840

Population Bulletin, [s.l.], [s.n.], 19??-, P 1432

Population et avenir, Paris, [s.n.], 1971-, P 3155-686

Population et sociétés : Bulletin mensuel d'informations démographiques, économiques et sociales, Paris, Institut national d'études démographiques, 1968-, P 3045

Portraits d'économistes..., Paris, La Documentation française, 2007, P 1102-2934

Post-Soviet affairs, Silver Spring, MD, V.H. Winston \& Son, Inc, P 4320

Post-Soviet geography and economics, Palm Beach, FL, V.H. Winston and Son, [1996]-c2002, P 1395

Problèmes d'Amérique latine, Paris ; Paris ; [Paris] ; [Paris] ; [Paris], La Documentation française : La Documentation française : IEG, Institut européen de géoéconomie : Institut Choiseul pour la politique internationale et la géoéconomie : Choiseul, 1965-, $\mathbf{P}$ 4389

Problèmes économiques : sélection de textes français et étrangers, Paris, Documentation française, 1948-, P 1102

Problèmes politiques et sociaux : articles et documents d'actualité mondiale, Paris, Documentation française, 1970-, P 3367

Promet, Zagreb ; Portorož ; Trieste, Fakultet prometnih znanosti : Fakulteta za pomorstvo in promet : Universita degli studi di Trieste, Istituto per lo studio dei trasporti nell'intergrazione economica europea, $\mathbf{P} 4584$

PROOST, Transmission reprise de très petites entreprises du secteur marchand en Îlede-France, Paris, Conseil économique et social, 2007, P 4566 [88]

PY, Pierre, Le tourisme : un phénomène économique, Paris, la Documentation française, DL 2007, P 797-5261

Rapport, Paris, Conseil économique et social de la région d'Ile-de-France, 1994-..., P 4566

Raumforschung und Raumordnung, Köln ; Berlin ; Bonn ; München, Heymann, 1936-, P 1183

Regards croisés sur l'intégration européenne (1957-2007), Paris, A. Colin, 2007, P 497/ A-71-4

Religions, pouvoir et société : Europe centrale, Balkans, CEI, Paris, la Documentation française, 2004, P 3379-1045

REM. Revue de l'économie méridionale, Montpellier, Centre régional de la productivité et des études économiques, 1987-, P 1774

Revue d'écologie, Paris, Société nationale de protection de la nature et d'acclimatation de France, 1980-, P 435 

3379-1025 A-HS\%07

Revue d'économie régionale et urbaine, Paris ; Paris ; Paris, Editions Economica : Editions Economica : A. Colin, 1978-, P 3660-4

Revue de géographie alpine, Grenoble ; Grenoble ; Paris, Université de Grenoble : Université de Grenoble : A. Colin, 1920-, P 505

Revue de l'énergie, Paris, Ed. techniques et économiques, 1974-, P 1259

Revue du marché commun, Paris, Editions techniques et économiques, 1958-, P 1994

Revue Tiers monde, Paris ; Paris ; Paris, Presses universitaires de France : Presses universitaires de France : A. Colin, 1997-, P 1414

RIBEIRO, Finisterra : revista portuguesa de geografia, Lisboa, Centro de Estudos Geográficos, 1966-, P 1858-42

ROBERT, SCHAEFFER, Modes de vie et identités franciliennes, aujourd'hui et demain, Paris, CESR Île-de-France, 2007, P 4566-89

RURDS. Review of urban and regional development studies, Tokyo ; Tokyo, Applied Regional Science Conference : Tokyo International University, 1989-..., P 4592

Russie : bilan du premier mandat de Vladimir Poutine, Paris, la Documentation française, 2003, P 3379-1038

Russie : l'armée en mutation, Paris, la Documentation française, 2002, P 3379-1022

Russie-Europe : vers quelle intégration ?, Paris, la Documentation française, 2002, P

RUSTEN, HERMELIN, The geography of services, Stockholm, Svenska sällskapet för antropologi och geografi, 2007, P 99/C-89-1\%07

SCHÖLZEL, Christian, Palaeoenvironmental transfer functions in a Bayesian framework with application to holocene climate variability in the Near East, Sankt Augustin, Asgard-Verlag, 2006, PCD 29-62

Sciences humaines, Auxerre, Sciences humaines, 1989-, P 4384

Ségrégation urbaine et politiques publiques en Europe, Paris, I.A.U.R.I.F, 2003, P 2268/

SEMMEL, Arno, Die Erde im Frankfurter Stadtwald, Frankfurt, M., Inst. für Physische Geographie, 2006, P 3812-29

SHARP, Papers from the International Symposium on Cryospheric Indicators of Global Climate Change, held in Cambridge, UK, on 21-25 August 2006, Cambridge, UK, International Glaciological Society, c2007, P 3791-46

SIG la lettre, Saint-Quentin-en-Yvelines, Rouge vif, 1998-, P 4571

SMEDT, Les Cahiers d'espaces, Paris, Editions touristiques européennes, 1985-, P 4198

Sociaux-démocrates et populistes dans la nouvelle Europe, Paris, La Documentation française, DL 2006, P 3379-1054

STARK, Alexander, Wirtschaftsförderung und "Good Governance“ in Argentinien : Ansätze für eine dynamische Regionalentwicklung, Tübingen, Geographisches Institut der Universität Tübingen, 2007, P 1728-144

Tableaux économiques de l'Auvergne, Chamalière, INSEE Auvergne, DL 2007,

P 4666\%07/\%08

Tableaux économiques de l'Auvergne, Chamalières, INSEE Auvergne, 2003-, P 4666-2007 

1288-109 1965-, P 1177 1670-79-1

Tagungsband zum Workshop über den Wissenschaftlichen Erkenntnisstand über das Feinstaubfilterungspotenzial (Qualitativ und Quantitativ) von Pflanzen am 1. Juni in Berlin-Adlershof , Berlin, Geographisches Institut der Humboldt-Universität, 2007, P test périodiques formation juin, Paris, institut de geo, P 10000-4/6 The California geographer, [Long Beach, Calif.?, [s.n.], 1960-, P 1706-47

The Journal of geology, Chicago, IL, University of Chicago Press, 1893-, P 239

The Statesman's year-book, New York, St. Martin's Press, 1864-, P 128

TIRONE, Lucien, La région Provence-Alpes-Côte d'Azur à l'aube du XXIe siècle, Aix-enProvence, Association des « Amis de la Revue Méditerranée » : Institut de géographie de l'Université de Provence, 2003, P 1420-101

Town \& country planning, London, Town and Country Planning Assoc, P1876

Town planning review, Liverpool, Liverpool University Press, 1910-, P 1849

Transactions Institute of British Geographers, London, Institute of British Geographers,

TRÉGOUËT, NIRASCOU, Le 4 pages : lettre thématique mensuelle de l'Institut français de l'environnement, Orléans, IFEN, 2005-, P 4691/A

Urban geography, Silver Spring, Md, V.H. Winston, P 3839

Vegetation maps as a tool in environmental assessment and spatial planning, Warszawa, Polish Academy of Sciences. Institute of Geography and Spatial Organization, 2006, P

Vie universitaire : le mensuel de l'enseignement supérieur, Paris, Les Ed. de la Vie universitaire, 1997-, P 4594

Ville \& transports : le magazine professionnel du transport européen, Paris, La Vie du rail, 2005-, P 4680

Villes, territoires, mondialisation, Paris, A. Colin, 2007, P 3660\%07-4

Vodnye resursy, Moskva, Nauka, 1972-, P 3646

WANG, Hui, The forefront of urban China : new special development zones and their impact on the spatial transformation of Chinese cities : a case study of Xi'an, Köln, Geographisches Institut der Universität zu Köln, 2007, P 1153-88

Water resources research, Washington [etc.], American Geophysical Union, 1965-, P 1885

WIEGANDT, Beiträge zum Festkolloquium aus Anlass der Benennung des Hörsaals des Geographischen Instituts in „Alfred-Philippson-Hörsaal“, Sankt Augustin, AsgardVerlag, 2007, P 3914-29

Wirtschaft und Statistik, Wiesbaden, Statistisches Bundesamt, P 5

Witterung in Übersee, Hamburg, Deutsches Seewetteramt, P 4438-55-1

XYZ : la revue de l'Association française de topographie, Paris, Association française de topographie, 1979-, P 4478

Zeitschrift für Wirtschaftsgeographie, Frankfurt, Buchenverl, 1957-, P 1376

ZIMMERMANN, Michael, Spectroscopic quantification of soil organic carbon fractions that can be related to model pools, Zürich, Geographisches Institut der Universität, cop. 
2007,

P 3881-56

ZUBAKOV, Noveïseï ètap : pozdniï pliocen - ?etverti?nyï period, Leningrad, Nedra, 1974,

R 851

Répertoires, dictionnaires

574 BRUNET, Jérôme, Assistant des bibliothèques : catégorie B, Paris, Vuibert, impr. 2007, G 1-814

Environnement et aménagement : cartes utiles, Paris-La Défense, Ed. du STU, 1995, G 1-637-<1>

576 JOLY, Gérard, Répertoire des géographes français 2007, Paris, CNRS-UMR-PRODIG, 2007, G 1-759

LAPERGUE, Maryse, SERRE, Le développement durable de A à Z, Baixas ; Paris, Balzac : Nérée, cop. 2007, W 359

Mener un projet Open Source en bibliothèque, documentation et archives, Paris, Ed. du Cercle de la librairie, DL 2007, G 1-812

MOUREN, Manuel du patrimoine en bibliothèque, Paris, Éd. du Cercle de la librairie, DL 2007, G 1-811

RIVIER, Alexis, Aide-mémoire d'informatique documentaire, Paris, Éd. du Cercle de la librairie, DL 2007, G 1-813

VYRET-MEKDJIAN, Dictionnaire de l'environnement, Paris, A. Colin, DL 2007, W 357

Théorie - Enseignement - Méthodes - Cartographie

BERTIN, Jacques, La graphique et le traitement graphique de l'information, Paris, Flammarion, imp.1977, G 5-754

BLANC, Jean-Noël, CHÉNOT, Le projet et la « pensée projet », Saint-Etienne, Publications de l'Université de Saint-Etienne, DL 2007, G 5-1682

CAUVIN, Colette, Cartographie thématique, Paris, Hermes science : Lavoisier, impr. 2007, G 5-1677-<1>

DAINVILLE, François de, La géographie des humanistes, Paris, Beauchesne, 1940, G 5-181

HUBBARD, KITCHIN, VALENTINE, Key thinkers on space and place, London ; Thousand Oaks (Calif.) ; New Delhi, Sage publications, cop. 2004, G 5-1692

587 JAKOB, Michael, Paysage et temps : comment sortir du musée du paysage contemporain, Gollion, Infolio éditions, cop. 2007, G 5-1684

JUILLARD, Étienne, La Région : contributions à une géographie générale des espaces régionaux, Paris, Éditions Ophrys, 1974, G 5-901 L'orbite de la géographie de Jean Gottmann : [actes du colloque international qui s'est tenu à Paris les 29 et 30 mars 2005], Paris, Société de géographie, impr. 2006, G 5-1643 LATOUR, Bruno, BIEZUNSKI, La vie de laboratoire : la production des faits scientifiques, Paris, la Découverte, impr. 2006, G 5-1681

591 Le développement durable : une idéologie ?, G 5-1698

592 LECOQ, CHAMBARD, Terre à découvrir, terres à parcourir, Paris, Publications de l'Université Paris 7-Denis Diderot, 1996, A 5.1 79 
LEFEBVRE, Henri, ELDEN, LEBAS, KOFMAN, Key writings, New York ; London, Continuum, cop. 2003, G 5-1691

PEZEU-MASSABUAU, Jacques, Construire l'espace habité : l'architecture en mouvement, [Paris], l'Harmattan, DL 2007, G 5-1532

RACINE, Jean-Bernard, L'analyse quantitative en géographie, [Paris], Presses universitaires de France, 1973, G 5-665

SAID, Edward Wadie, MALAMOUD, MEININGER, TODOROV, WAUTHIER, L'orientalisme : l'Orient créé par l'Occident, Paris, Ed. du Seuil, impr. 2005, G 5-1680

SORRE, La Géographie..., Paris, Bourrelier, 1959, G 5-1686

WORSTER, Donald, A river running west : the life of John Wesley Powell, Oxford ; New York, Oxford University Press, cop. 2001, G 5-1693

Thèses

BORDES, Claire, PITTE, La construction de la défense européenne via l'élargissement à l'est de l'Union Européenne et de l'OTAN : complémentarité ou rivalité au sein de la nouvelle donne géopolitique européenne : thèse pour l'obtention du grade de docteur de l'Université Paris IV, [S.l.], [s.n.], 2007, T 2024

GIUSTI, Christian, CALVET, Le sud du Massif Central (France) : implications morphogénétiques de l'activation d'une marge passive : approches épistémologique et naturaliste, [S. 1.], [s. n.], 2002, T 1918-<1>

MESSAADI, Beya, CARMONA, Législation de l'urbanisme en Algérie : mythe ou réalité, S.l., s.n., 2006, T 2022-<1>

PINCENT, Guillemette, CARMONA, La réhabilitation des quartiers précoloniaux dans les villes d'Asie Centrale : étude de cas de Tachkent et de Boukhara (Ouzbékistan), [S.l.], [s.n.], 2007, T 2023-<1> 